\title{
Vibrational spectra of halophthalonitriles
}

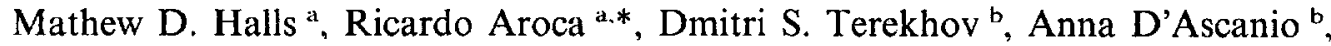 \\ Clifford C. Leznoff ${ }^{b}$ \\ "Materials and Surface Science Group. University of Windsor, Windsor, ON N9E3P4, Canada \\ ' Department of Chemistry, York Unitersity, North York, Toronto, ON M3JIP3. Canada
}

\begin{abstract}
The fundamental vibrational modes of a series of six halophthalonitriles have been studied using Raman and infrared spectroscopy. The vibrational assignment of experimental wave numbers obtained from solid samples was aided using quantum chemical computations. Semi-empirical methods and the local SVWN functional were used to obtain vibrational wave numbers and atomic displacement representations of the fundamental molecular vibrations. The study of a series of molecules with similar structure permits the identification of characteristic wave numbers and the effect of the halosubstitution in the molecular structure. C 1998 Elsevier Science B.V. All rights reserved.
\end{abstract}

Keywords: Raman; Infrared; Vibrations; Substituted phthalonitriles; Assignments; SVWN DFT

\section{Introduction}

In recent years the synthesis of near infrared absorbing dyes has been an increasingly active field. Phthalonitriles (1,2-dicyanobenzenes) are used as synthetic precursors for large macrocyclic organic semiconductors. particularly for phthalocyanines $[1,2]$ with strong absorption in the near infrared region of the spectrum. For the synthesis of substituted phthalocyanines, halogen substituted phthalonitriles are used. Vibrational studies of these precursor molecules are of interest in their own right and they are extremely valuable when performing vibrational studies of larger composite molecular systems. In the present work

* Corresponding author. a vibrational study of novel materials, a series of six halophthalonitriles, is presented: 3-iodophthalonitrile, 4-iodophthalonitrile, 4,5-diiodophthalonitrile, 4-bromophthalonitrile, 4,5-dibromophthalonitrile and 3,6-dibromophthalonitrile. Several vibrational studies of different phthalonitriles have been carried out [3-7]. However, the infrared and Raman spectra of the halophthalonitriles in this series have not been previously reported. The vibrational assignment of this series based on the FT-Raman and FT-infrared spectra is presented here for the first time. Of great assistance in spectral interpretation are quantum chemical calculations of theoretical harmonic fundamentals, atomic displacement representations and spectral intensities. It has become standard to perform these calculations using Hartree-Fock 
(HF), and the level of theory is largely determined by the computational capabilities. Usually $\mathrm{HF}$ based semi-empirical methods, such as AM1 and PM3, are used due to affordability and some previous knowledge of relative performance. In recent years new computational schemes have arrived which are known collectively as density functional models (DFT). Experience with DFT models is still relatively limited, however an increasing number of studies are being reported which show DFT models offer many desirable features, such as more favorable scaling with respect to system size and better performance than HF and post-HF (MP2) methods for calculating frequencies and infrared intensities [8-10]. Presented in this report are proposed general vibrational assignments based on calculations performed using the semi-empirical AM1 and PM3 methods and the DFT SVWN local spin density functional.

\section{Experimental}

\subsection{Synthesis}

\subsubsection{3-Iodophthalonitrile}

The synthesis of 3-iodophthalonitrile was performed starting with 3-nitrophthalonitrile which was prepared using the procedure of Campagna et al. [11] in $95 \%$ yield. 3-Nitrophthalonitrile was selectively reduced to 3-aminophthalonitrile and was purified by flash chromatography in $83 \%$ yield. 3-Aminophthalonitrile was then acidified and treated with sodium nitrite to give the diazonium salt. The diazonium salt solution was added to a potassium iodide solution in water, resulting in 3-iodophthalonitrile. The aqueous product 3 . iodophthalonitrile was washed in benzene with $5 \% \mathrm{NaHCO}_{3}$, sat. $\mathrm{Na}_{2} \mathrm{~S}_{2} \mathrm{O}_{3}$, and cold water. The benzene solution was dried over anhydrous magnesium sulfate, and purified by chromatography on $200 \mathrm{~g}$ of normal grade silica gel in $62 \%$ yield [12].

\subsubsection{4-Iodophthalonitrile}

The synthesis of 4-iodophthalonitrile was performed starting with 4-nitrophthalonitrile. Ac- cording to the procedure of Rasmussen et al. [13], the 4-nitrophthalonitrile was reduced by $10 \%$ palladium on charcoal in EtOH and hydrogenation at $415 \mathrm{kPa}$, to yield 4-aminophthalonitrile. The product was purified by chromatography on normal grade silica gel in $84 \%$ yield. 4-Aminophthalonitrile was then acidified, cooled and treated with sodium nitrite. The resulting product was rapidly filtered by suction filtration and the cool filtrate was added to a solution of potassium iodide in water, reacting to produce 4-iodophthalonitrile. The product precipitate was collected by suction filtration, washed with cold water and dissolved in benzene. The benzene solution was then washed with cold water. $5 \% \mathrm{NaHCO}_{3}$, sat. $\mathrm{Na}_{2} \mathrm{~S}_{2} \mathrm{O}_{3}$ and washed dried over $\mathrm{MgSO}_{4}$. Chromatography on $200 \mathrm{~g}$ of normal grade silica gel with benzene gave 4-iodophthalonitrile in $70 \%$ yield [14].

\subsubsection{5-Diiodophthalonitrile}

The synthesis of 4,5-diiodophthalonitrile was performed starting with phthalimide. To 30\% fuming sulfuric acid was added phthalimide and iodine. After heating for $24 \mathrm{~h}$ the mixture was cooled over ice and the precipitate was collected using a funnel and a glass frit. The solids were washed with water, $2 \% \mathrm{~K}_{2} \mathrm{CO}_{3}$, sat. $\mathrm{Na}_{3} \mathrm{~S}_{2} \mathrm{O}_{3}$ and were dried at room temperature. The solids were extracted with acetone in a Soxhlet extractor for $48 \mathrm{~h}$. The resulting solid was 4,5-diiodophthalic acid. The 4,5-diiodophthalic acid was filtered from the acetone and water was added. The solution was concentrated and cooled to give a precipitate of 4,5-diiodophthalimide. Chromatography on silica gel using $\mathrm{CHCl}_{3}-\mathrm{EtAC}$ as eluent gave an isolated yield of $75 \%$. To concentrated aqueous ammonia, pure 4,5-diiodophthalimide was added. This mixture was then stirred and heated to 50 -$60^{\circ} \mathrm{C}$ for $1.5 \mathrm{~h}$, to give a solid intermediate. The solid was filtered and washed with cold water and methanol, and was then dried at room temperature to give 4,5 -diiodophthamide in $81 \%$. 4,5-Diiodophthamide was then suspended in ice-cooled dry dioxane and dry pyridine and trifluoroacetic anhydride was added at $0-5^{\circ} \mathrm{C}$. After the addition the reaction mixture was warmed to room temperature and poured on ice. The product was ex- 


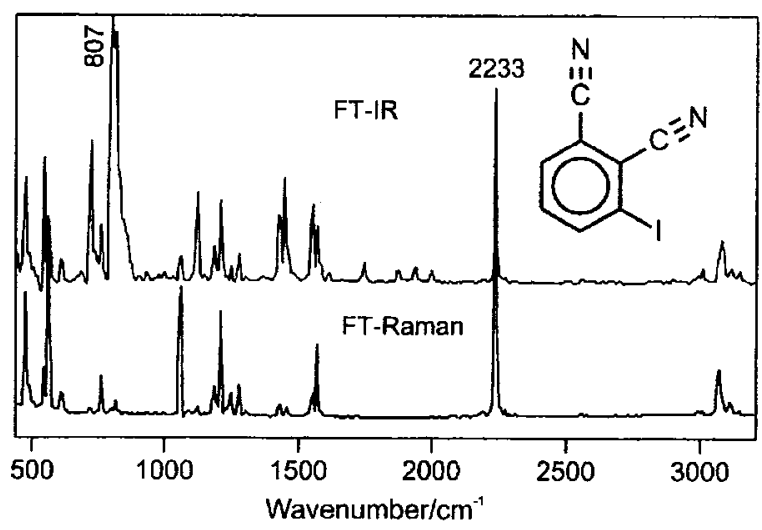

Fig. 1. FT-IR and FT-Raman spectra of 3-iodophthalonitrile.

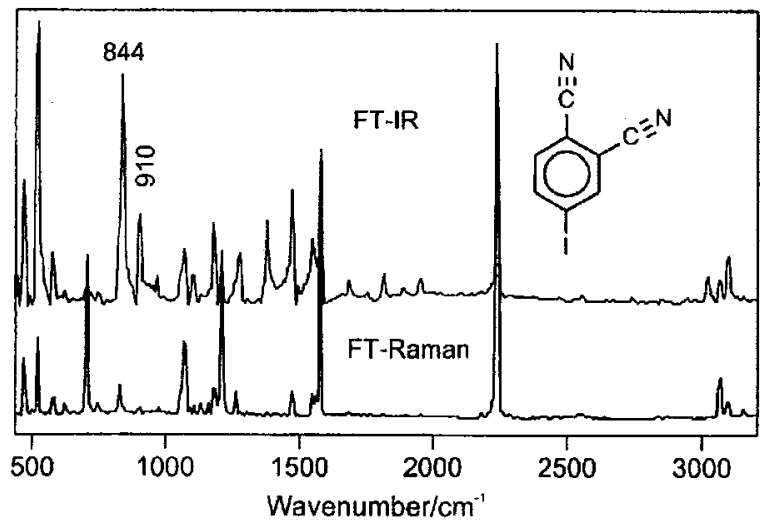

Fig. 2. FT-IR and FT-Raman spectra of 4-iodophthalonitrile.

tracted with EtOAC. The organic layer was washed with water, $\mathrm{HCl}$, dilute $\mathrm{Na}_{2} \mathrm{CO}_{3}$ and was dried over $\mathrm{MgSO}_{4}$. The product was recrystallized from ethanol to give 4-iodophthalonitrile in $79 \%$ yield [15].

\subsubsection{4-Bromophthalonitrile, 4,5-dibromo-} phthalonitrile and 3,6-dibromophthalonitrile

Dibromoisocyanuric acid was prepared by the procedure of Gottardi [16]. Dibromoisocyanuric acid was dissolved in $8 \%$ fuming sulfuric acid at room temperature. The solution was cooled in ice and phthalonitrile (1,2-dicyanobenzene) was added to solution. The mixture was stirred and then poured into ice water. The resultant mixture was then extracted with ether and the extract was washed with $\mathrm{H}_{2} \mathrm{O}, 1 \% \mathrm{NaHCO}_{3}, 1 \% \mathrm{NaHSO}_{3}$. and dried over anhydrous $\mathrm{MgSO}_{4}$. Evaporation gave a solid mixture which was separated on a silica gel column using ethyl acetate-hexane as eluent to give products 4-bromophthalonitrile $45.2 \%, 4,5$-dibromophthalonitrile $6.7 \%$ and 3,6-dibromophthalonitrile $7 \%$ [17].

\subsection{Infrared and Raman spectra}

The infrared spectra of all molecules in this study were collected from CsI pellets using a 


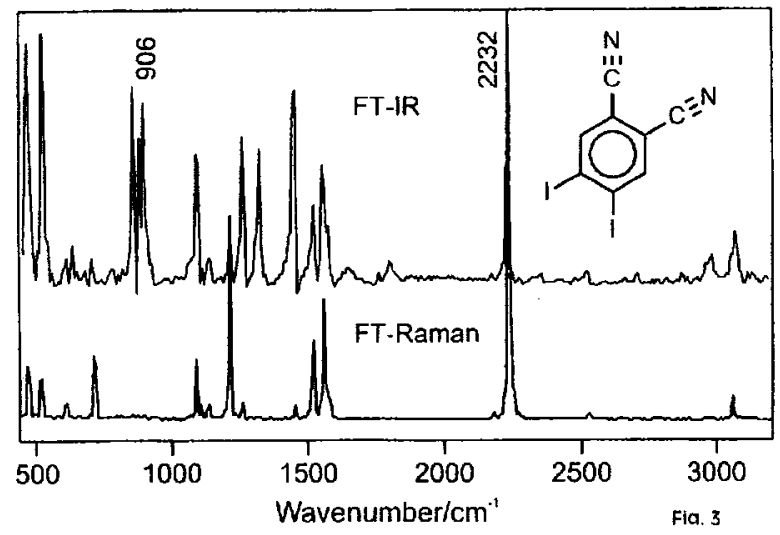

Fig. 3. FT-IR and FT-Raman spectra of 4,5-diiodophthalonitrile.

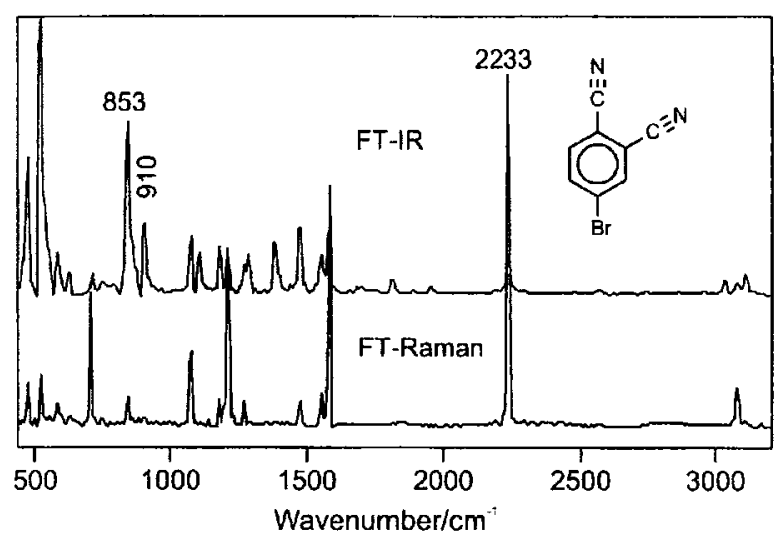

Fig. 4. FT-IR and FT-Raman spectra of 4-bromophthalonitrile.

Bomem DA3 FTIR spectrometer with far-infrared and mid-infrared accessories. A KBr beam splitter and liquid nitrogen cooled MCT detector were used to collect the mid-infrared spectra from 4000 to $400 \mathrm{~cm}$ '. A $6 \mathrm{~mm}$ stretched Mylar pellicle beam splitter and a DTGS detector were used to collect the far-infrared spectra from 500 to $100 \mathrm{~cm}^{-1}$. The FT-Raman spectra of the molecules in this study were collected from solid capillary using a Bomem Ramspec 150 spectrophotometer with an Nd:YAG laser emitting at $1064.1 \mathrm{~nm}$ and a InGaAs detector. The FT-Raman system with a Michelson interferometer is interfaced to a NEC powermate computer. The
FT-Raman spectra were collected from 3400 to $350 \mathrm{~cm}^{-1}$.

\subsection{Computational methods}

Semi-empirical calculations using the AM1 [18] and the PM3 [19] Hamiltonians have been performed on all molecules in this study. The semiempirical computations were performed on a 486 PC using Gaussian92, Revision E.1 [20]. DFT calculations using the SVWN local spin density functional Hamiltonian $[21,22]$ with a $\mathrm{DN}^{* *}$ basis set (numerical split valence basis set supplemented by d-type functions on heavy atoms and p-type 


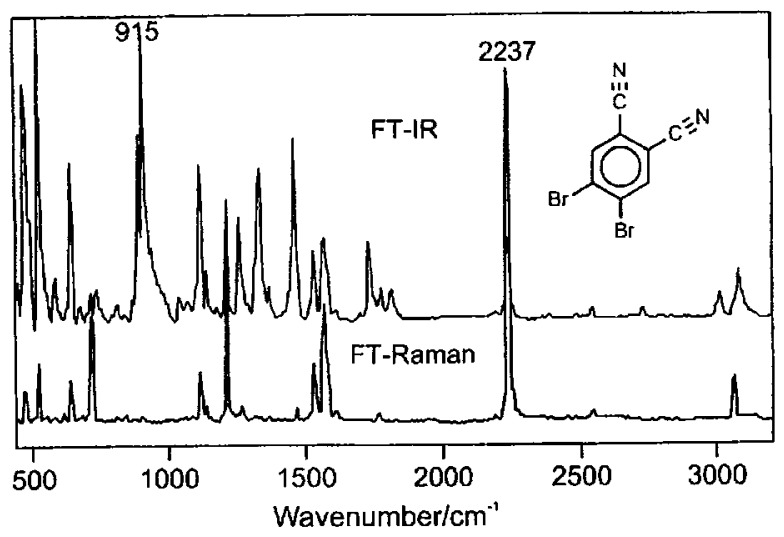

Fig. 5. FT-IR and FT-Raman spectra of 4,5-dibromophthalonitrile.

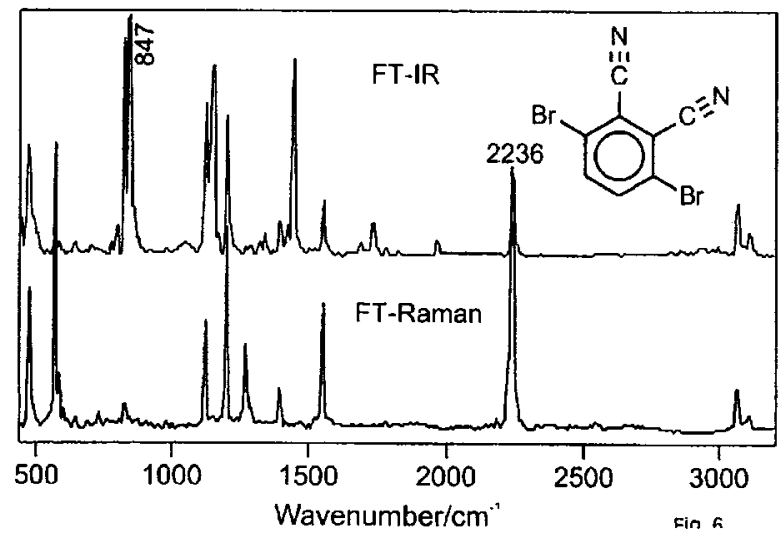

Fig. 6. FT-IR and FT-Raman spectra of 3,6-dibromophthatonitrile.

functions on hydrogens) were performed for all bromo-substituted phthalonitriles in this study. The DFT calculations were performed on a RS6000 workstation using Spartan IBM V.4.1.2GL [23]. All calculations involved first geometry optimization and subsequent harmonic frequency calculation at the same level of theory.

\section{Results and discussion}

\subsection{Molecular structure and symmetry}

The substituted phthalonitriles in this study correspond to 3-iodophthalonitrile, 4-iodo- phthalonitrile, 4,5-diiodophthalonitrile, 4-bromophthalonitrile, 4,5-dibromophthalonitrile and 3,6dibromophthalonitrile. Geometry optimizations at AM1, PM3 and SVWN levels of theory predict planar geometries for the molecules considered here. The molecular series considered here can be divided into molecules belonging to one of two symmetry point groups: $C_{s}$ or $C_{2 r}$. Apart from the identity element, phthalonitriles of $C_{s}$ symmetry only have the molecular plane as a symmetry element. The $C$, molecules include all singly substituted phthalonitriles in this series: 3-iodo-, 4-iodo-, 4-bromo-. Phthalonitriles of $C_{2 x}$ symmetry have a 2 -fold rotational axis and an 
Table 1

Symmetry of vibration. AM1. PM3, SVWN, experimental wavenumbers ( $\mathrm{cm}^{-1}$ ), and band assignments for 4-bromophthalonitrile

\begin{tabular}{|c|c|c|c|c|c|c|c|c|c|}
\hline Mode & Sym & AMI & PM3 & SVWN & AM1 & PM3 & SVWN & Observed & Assignment \\
\hline 1 & $a^{\circ}$ & 72 & 71 & 66 & 64 & 63 & 6.5 & & $\mathrm{Br} w a g+C \mathrm{CN}$ wag \\
\hline 2 & $a^{\prime}$ & 123 & 124 & 132 & 110 & 110 & 130 & & $\mathrm{CBr}$ bend $+\mathrm{CCN}$ bend \\
\hline 3 & $a$ & 1,32 & 126 & 137 & 118 & 113 & 134 & & CCN wag \\
\hline 6 & $a:$ & 291 & 270 & 276 & 259 & 240 & 271 & & $\mathrm{CBr}$ stretch $+\mathrm{CCN}$ bend \\
\hline 7 & $a^{\prime}$ & 299 & 288 & 280 & 266 & 256 & 276 & 267 & $\mathrm{CBr}$ bend $+\mathrm{CCN}$ bend \\
\hline 8 & $a^{\prime \prime}$ & 377 & 364 & 372 & 336 & 324 & 365 & 374 & Ring torsion + CCN wag \\
\hline 9 & $a^{\prime \prime}$ & 4.37 & 404 & 409 & 389 & 360 & 402 & & $\mathrm{CCN}$ torsion + ring torsion $+\mathrm{CH}$ wag \\
\hline 10 & $a^{:}$ & 489 & 63 & 430 & 435 & 412 & 422 & 4.39 & Ring deformation \\
\hline 15 & $a^{\prime}$ & 718 & 662 & 642 & 639 & 589 & 631 & 634 & CCN bend \\
\hline 16 & $a^{\prime \prime}$ & 786 & 761 & 729 & 700 & 677 & 717 & 717 & $\mathrm{CCN}$ torsion $+\mathrm{CH}$ wag \\
\hline 17 & $a^{\prime}$ & 876 & 828 & 730 & 780 & 737 & 718 & 722 & Ring deformation \\
\hline 18 & $a^{: r}$ & 893 & 867 & 811 & 795 & 771 & 797 & & $\mathrm{CH}$ torsion \\
\hline 19 & $a^{t}$ & 936 & 908 & 857 & 833 & 808 & 84.3 & 841 & Ring deformation + CCN stretch \\
\hline 20 & $a^{\prime \prime}$ & 958 & 948 & 867 & 853 & 844 & 8.52 & 853 & $\mathrm{CH}$ wag \\
\hline 21 & $a^{\prime \prime}$ & 995 & 996 & 939 & 886 & 887 & 923 & 910 & $\mathrm{CH}$ wag \\
\hline 22 & $a^{\prime}$ & 1189 & 1088 & 1080 & 1058 & 968 & 1062 & 1079 & $\mathrm{CH}$ bend \\
\hline 23 & $a^{\prime}$ & 1203 & 1121 & 1112 & 1071 & 998 & 1094 & {$[1] 10$} & $\mathrm{CH}$ bend \\
\hline 24 & $a^{\prime}$ & 1292 & 1182 & 1192 & 1150 & 1052 & 1172 & 1180 & $\mathrm{CH}$ bend \\
\hline 25 & $a^{\prime}$ & 1329 & 1272 & 1226 & 1183 & 1132 & 1205 & 1211 & CC stretch + CCN stretch \\
\hline 34 & $a^{\prime}$ & 3167 & 3054 & 3135 & 2819 & 2718 & 30182 & 3034 & $\mathrm{CH}$ stretch \\
\hline 35 & $a^{\prime}$ & 3169 & 3061 & 3157 & 2820 & 2724 & 3105 & 3074 & CH stretch \\
\hline 36 & $a^{\prime}$ & 3181 & 3069 & 3170 & 2831 & 2731 & 3117 & 3105 & $\mathrm{CH}$ stretch \\
\hline
\end{tabular}

additional plane of symmetry. The $C_{21}$ molecules include all doubly substituted phthalonitriles in this series: 4,5-diiodo-4,5-dibromo-, 3,6-dibromo. With 14 atoms composing each structure, each molecule has 36 fundamental modes of vibration. For molecules of $C$, symmetry, the total irreducible representation of the 36 fundamentals is: $\Gamma=25 a^{\prime}+11 a^{\prime \prime}$, where all normal modes are in- frared and Raman active. For molecules of $C_{2 t}$ symmetry, group theory analysis indicates that the 36 fundamental vibrations will reduce as: $\Gamma=$ $13 a_{1}+6 a_{2}+5 b_{1}+12 b_{2}$. In the $C_{2 r}$ point group all normal vibrations are Raman active and all but the $a_{2}$ vibrations are infrared active. A final general comment about the normal vibrations of this series of molecules is that all phthalonitriles will 
Table 2

Symmetry of vibration, AMI, PM3, SVWN, experimental wavenumbers $\left(\mathrm{cm}^{-1}\right)$, and band assignments for 4,5-dibromophthalonitrile

\begin{tabular}{|c|c|c|c|c|c|c|c|c|c|}
\hline \multicolumn{2}{|c|}{ 4.5-Bromo } & \multicolumn{3}{|c|}{ Nonscaled } & \multicolumn{3}{|l|}{ Scaled } & \multirow[t]{2}{*}{ Observed } & \multirow[t]{2}{*}{ Assignment } \\
\hline Mode & Sym & $A M 1$ & PM3 & SVWN & AMI & PM3 & SVWN & & \\
\hline 1 & $a_{2}$ & 47 & 52 & 45 & 42 & 46 & 45 & & $\mathrm{CBr}+\mathrm{CCN}$ wag \\
\hline 2 & $b_{1}$ & 101 & 93 & 82 & 89 & 83 & 80 & & CBr wag \\
\hline 3 & $u_{1}$ & 117 & 125 & 122 & 104 & 111 & 120 & & $\mathrm{CBr}$ bend $+\mathrm{CCN}$ bend \\
\hline 4 & $a_{1}$ & 127 & 134 & 131 & 113 & 119 & 128 & & $\mathrm{CBr}$ bend $+\mathrm{CCN}$ bend \\
\hline 5 & $b_{2}$ & 143 & 160 & 141 & 127 & 143 & 139 & 146 & $\mathrm{CBr}$ rock $+\mathrm{CCN}$ rock \\
\hline 6 & $a_{3}$ & 164 & 178 & 153 & 146 & 158 & 151 & 166 & $\mathrm{CCN}$ wag + CBr wag \\
\hline 7 & $b_{2}$ & 263 & 248 & 239 & 234 & 221 & 235 & 214 & CBr stretch \\
\hline 8 & $b_{1}$ & 280 & 253 & 252 & 249 & 225 & 248 & 242 & $\mathrm{CBr}+\mathrm{CCN}$ wag \\
\hline 9 & $a_{1}$ & 335 & .326 & 306 & 298 & 290 & 301 & 269 & $\mathrm{CBr}$ stretch + ring deformation \\
\hline 10 & $b_{2}$ & 359 & 340 & 332 & 320 & 302 & 326 & 337 & $\mathrm{CCN}$ bend $+\mathrm{CBr}$ bend \\
\hline 11 & $b_{1}$ & 422 & 384 & 391 & 376 & 342 & 385 & & Ring torsion $+\mathrm{CH}$ wag \\
\hline 12 & $a_{2}$ & 441 & 412 & 416 & 392 & 367 & 409 & 413 & CCN torsion + ring torsion \\
\hline 13 & $b_{2}$ & 5.32 & 500 & 480 & 473 & 445 & 472 & 480 & Ring deformation \\
\hline 14 & $a_{1}$ & 583 & 543 & 497 & 519 & 483 & 488 & 494 & $\mathrm{CCN}$ bend \\
\hline 15 & $b_{1}$ & 625 & 579 & 519 & 556 & 515 & 510 & 534 & $\mathrm{CCN}$ wag $+\mathrm{CH}$ wag \\
\hline 16 & $a_{2}$ & 695 & 654 & 636 & 618 & 582 & 626 & 589 & Ring torsion \\
\hline 17 & $a_{1}$ & 703 & 668 & 643 & 626 & $\$ 94$ & 632 & 627 & Ring deformation \\
\hline 18 & $b_{2}$ & 731 & 706 & 654 & 651 & 629 & 64.3 & 646 & $\mathrm{CCN}$ bend \\
\hline 19 & $a_{2}$ & 785 & 763 & 721 & 699 & 679 & 709 & 712 & CCN torsion \\
\hline 20 & $a_{1}$ & 894 & 837 & 739 & 796 & 745 & 727 & 723 & Ring stretch $+C C^{\prime} N$ stretch \\
\hline 21 & $b_{1}$ & 951 & 938 & 868 & 846 & 835 & 853 & 888 & $\mathrm{CH}$ wag \\
\hline 22 & $b_{2}$ & 961 & 954 & 876 & 856 & 849 & 861 & 899 & Ring deformation \\
\hline 23 & $a_{2}$ & 980 & 960 & 899 & 872 & 854 & 884 & 915 & $\mathrm{CH}$ wag \\
\hline 24 & $a_{1}$ & 12017 & 1091 & 1109 & 1074 & $97 !$ & 1091 & 1119 & $\mathrm{CH}$ bend \\
\hline 25 & $b_{2}$ & 1281 & 1144 & 1158 & 1140 & 1018 & 11.39 & 1146 & $\mathrm{CH}$ bend \\
\hline 26 & $b_{2}$ & 1322 & 126.3 & 1222 & 1176 & 1124 & 1201 & 1220 & Ring deformation \\
\hline 27 & $a_{1}$ & 1.357 & 13.34 & 1236 & 1208 & 1187 & 1216 & 1267 & $C \mathrm{C}$ stretch \\
\hline 28 & $a_{1}$ & 1454 & 1455 & 1365 & 1294 & 1295 & 1342 & 1324 & Ring stretch \\
\hline 29 & $h_{2}$ & 1549 & 1571 & 1375 & 1379 & 1399 & 1352 & 1336 & $C C$ stretch \\
\hline 30 & $a_{1}$ & 1646 & 1619 & 1473 & 1465 & $144 \mid$ & 1449 & 1466 & CC stretch \\
\hline 31 & $a_{1}$ & 1742 & 1786 & 1551 & 1550 & 1589 & 1525 & 1529 & CC stretch \\
\hline 32 & $b_{2}$ & 1765 & 1805 & 1604 & 1571 & 1607 & 1577 & 1566 & CC stretch \\
\hline 33 & $b_{2}$ & 2560 & 2474 & 2295 & 2278 & 2202 & 2256 & 2237 & CN stretch \\
\hline 34 & $a_{1}$ & 2560 & 2475 & 2299 & 2279 & 2202 & 2260 & 2237 & CN stretch \\
\hline 35 & $h_{2}$ & 3161 & 3064 & 3141 & 2814 & 2727 & 3083 & 3013 & $\mathrm{CH}$ stretch \\
\hline 36 & $a_{1}$ & 3164 & 3065 & 3174 & 2816 & 2728 & 3121 & 3087 & CH stretch (sym) \\
\hline
\end{tabular}

have 11 out-of-plane vibrations, 14 stretching vibrations and 11 in-plane bending vibrations.

\subsection{Assignment of spectra and effect of halosubstituent}

The structures, FT-Raman and infrared spectra of halophthalonitriles are shown in Figs. 1-6. Experimental wave numbers, symmetry of vibra- tion, theoretical frequencies and proposed general assignments for each of these molecules are presented in Tables 1-6. Frequencies are reported in wave numbers $\left(\mathrm{cm}^{\mathrm{l}}\right)$. When using computational methods to predict theoretical normal vibrations for relatively complex polyatomics, scaling strategies are used to bring computed wave numbers into closer agreement with observed frequencies. For the semi-empirical and 
Table 3

Symmetry of vibration, AM1. PM3, SVWN, experimental wavenumbers $\left(\mathrm{cm}^{-1}\right)$, and band assignments for 3,6-dibromophthalonitrile

\begin{tabular}{|c|c|c|c|c|c|c|c|c|c|}
\hline \multicolumn{2}{|c|}{ 3,6-Bromo } & \multicolumn{3}{|c|}{ Nonscaled } & \multicolumn{3}{|c|}{ Scaled } & \multirow[t]{2}{*}{ Observed } & \multirow[t]{2}{*}{ Assignment } \\
\hline Mode & Sym & AM1 & PM3 & SVWN & AMl & PM3 & SVWN & & \\
\hline 1 & $a_{2}$ & 65 & 62 & 72 & 57 & 55 & 71 & & CCN wag \\
\hline 2 & $b_{i}$ & 66 & 68 & 79 & 58 & 61 & 77 & & $\mathrm{CBr}$ wag \\
\hline 3 & $b_{2}$ & 129 & 99 & 122 & 115 & 88 & 120 & & CCN rock \\
\hline 4 & $a_{1}$ & 1.31 & 105 & 137 & 116 & 94 & 135 & & $\mathrm{CCN}$ bend \\
\hline 5 & $a_{1}$ & 151 & 140 & 150 & 134 & 125 & 147 & 147 & $\mathrm{CBr}$ bend $+\mathrm{CCN}$ bend \\
\hline 6 & $b_{1}$ & 190 & 173 & 177 & 169 & 154 & 174 & 180 & CCN wag \\
\hline 7 & $a_{1}$ & 230 & 222 & 217 & 205 & 197 & 213 & & Ring deformation \\
\hline 8 & $a_{2}$ & 268 & 247 & 264 & 239 & 220 & 259 & & $\mathrm{CBr}$ wag \\
\hline 9 & $b_{2}$ & 342 & 303 & 310 & 304 & 270 & 305 & 303 & $\mathrm{CBr}$ bend $+\mathrm{CCN}$ bend \\
\hline 10 & $a_{2}$ & 387 & 370 & 360 & 345 & 330 & 354 & & $\mathrm{CCN}$ torsion $+\mathrm{CH}$ torsion \\
\hline 11 & $b_{2}$ & 388 & 370 & 370 & 346 & 330 & 364 & 359 & $\mathrm{CBr}$ stretch + ring deformation \\
\hline 12 & $b_{1}$ & 499 & 452 & 432 & 444 & 402 & 425 & 441 & $\mathrm{CCN}$ wag + ring torsion \\
\hline 1.3 & $b_{2}$ & 526 & 503 & 483 & 468 & 447 & 475 & 478 & $\mathrm{CBr}$ stretch \\
\hline 14 & $a_{i}$ & 589 & 5.31 & 494 & 524 & 472 & 485 & 488 & CCN bend \\
\hline 15 & $a_{2}$ & 595 & 559 & 566 & 529 & 498 & 556 & 560 & Ring torsion + CCN wag \\
\hline 16 & $a_{1}$ & 670 & 643 & 589 & 596 & 572 & 580 & 577 & Ring deformation $+\mathrm{CCN}$ bend \\
\hline $1 ?$ & $b_{1}$ & 692 & 646 & 601 & 616 & 575 & $59 !$ & 591 & CCN wag \\
\hline 18 & $b_{2}$ & 734 & 668 & 653 & 653 & 595 & 642 & 650 & $\mathrm{CCN}$ bend \\
\hline 19 & $a_{2}$ & 8.33 & 828 & 756 & 742 & 737 & 744 & 7.34 & CCN torsion \\
\hline 20 & $b_{2}$ & 885 & 855 & 787 & 788 & 761 & 774 & 787 & Ring deformation \\
\hline 21 & $a_{1}$ & 922 & 895 & 840 & 821 & 796 & 826 & 832 & Ring deformation \\
\hline 22 & $b_{1}$ & 976 & 941 & 860 & 868 & 838 & 846 & 847 & CH wag \\
\hline 23 & $a_{2}$ & 996 & 994 & 929 & 886 & 884 & 914 & & CH wag \\
\hline 24 & $a_{1}$ & 1220 & 1119 & 1124 & 1086 & 996 & 1106 & 1127 & $\mathrm{CH}$ bend \\
\hline 25 & $b_{2}$ & 1237 & 1126 & 114.3 & 1101 & 1002 & 1124 & 1144 & $\mathrm{CH}$ bend \\
\hline 26 & $b_{2}$ & 1343 & 1295 & 1210 & 1196 & 1153 & 1189 & 1152 & Ring deformation. \\
\hline 27 & $\overline{a_{1}}$ & 1358 & 1338 & 1216 & 1209 & 1191 & 1195 & 1204 & CC siretch \\
\hline 28 & $a_{1}$ & 1424 & 1406 & 1372 & 1267 & 1251 & 1349 & 1270 & Ring stretch \\
\hline 29 & $b_{2}$ & 1582 & 1579 & 1417 & 1408 & 1405 & 1394 & 1395 & CC stretch \\
\hline 30 & $a_{i}$ & 1635 & 1641 & 1432 & 1455 & 1460 & 1409 & 1445 & CC stretch \\
\hline 31 & $a_{1}$ & 1742 & 1755 & 1571 & 1551 & 1562 & 1545 & 1556 & CC stretch \\
\hline 32 & $b_{2}$ & 1753 & 1824 & 1595 & 1560 & 1624 & 1568 & & CC stretch \\
\hline 33 & $b_{2}$ & 2558 & 2476 & 2295 & 2277 & 2204 & 2257 & 22.36 & CN stretch \\
\hline 34 & $a_{t}$ & 2559 & 2477 & 2301 & 2277 & 2204 & 2262 & 2236 & CN stretch \\
\hline 35 & $b_{2}$ & 3162 & 3058 & 3143 & 2814 & 2722 & 3090 & 3064 & $\mathrm{CH}$ stretch \\
\hline 36 & $a_{1}$ & 3173 & 3072 & 3156 & 2824 & 2734 & 3104 & 3107 & CH stretch (sym) \\
\hline
\end{tabular}

DFT methods employed in this work the simplest limiting scaling strategy was used - homogeneous scaling [24]. Semi-empirical fundamental frequencies were scaled by a factor of 0.89 . The SVWN local spin functional (Slater exchange functional [21] with VWN correlation functional [22]) has been shown to predict fundamental vibrations in good agreement with experimental data $[8,10]$. Recently, an empirical vibrational scaling factor for this functional has been reported to be 0.98 by Wong [9]. This empirical scaling factor was used to scale the computed SVWN fundamentals in an effort to increase the agreement between predicted and observed fundamentals. The average difference between unscaled frequencies and observed wave numbers for the semi-empirical AMI and PM3 methods were approximately 113 and 80 $\mathrm{cm}^{-1}$, respectively, across the entire series. The 
Table 4

Symmetry of vibration, AM1, PM3, experimental wavenumbers $\left(\mathrm{cm}^{-1}\right)$, and band assignments for 3-iodophthalonitrile

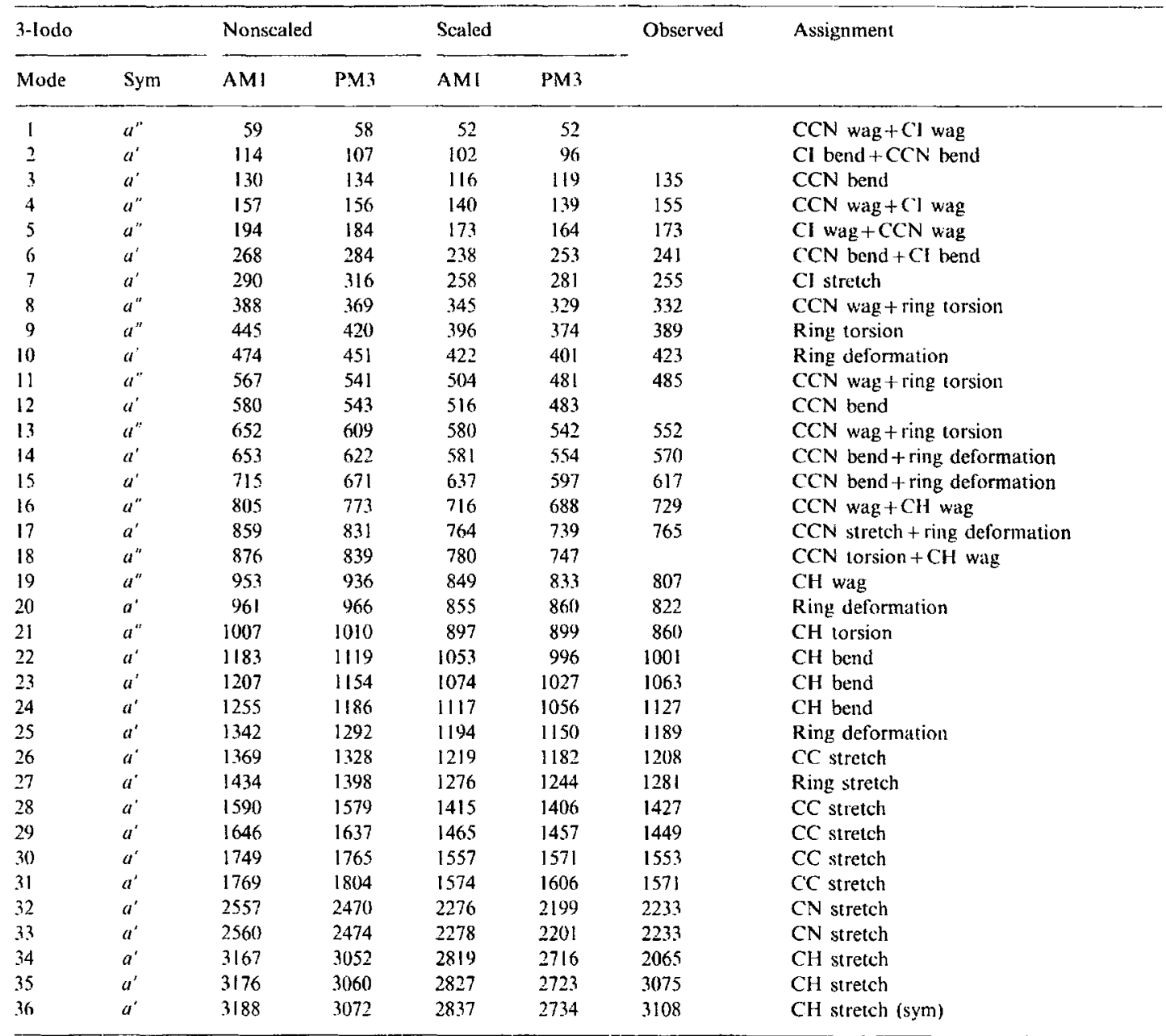

average difference for the SVWN functional was only ca. $27 \mathrm{~cm}^{-1}$, across the bromo-substituted phthalonitriles. All vibrational assignments are based on the respective point group symmetry for each molecule. Assignments were made through visualization of the atomic displacement representations for each vibration, and matching the predicted normal frequencies and intensities with experimental Raman and infrared data.
It is convenient to discuss the vibrational spectra of halophthalonitriles in terms of four characteristic spectral regions: $\mathrm{C}-\mathrm{H}$ stretching vibrations, $\mathrm{CN}$ stretching region, $\mathrm{C}=\mathrm{C}$ stretching and ring stretching modes region (1600-1000 $\left.\mathrm{cm}^{-1}\right)$, and out-of-plane $\mathrm{C}-\mathrm{H}$ vibrations region $\left(700-1000 \mathrm{~cm}^{-1}\right)$. The vibrational assignment for the phthalonitrile molecule (1,2-dicyanobenzene), aided with ab initio computations, has been carried out by Ramirez et al. [7]. The complete 
Table 5

Symmetry of vibration, AMI. PM3, experimental wavenumbers $\left(\mathrm{cm}^{-1}\right)$, and band assignments for 4-iodophthalonitrile

\begin{tabular}{|c|c|c|c|c|c|c|c|}
\hline \multicolumn{2}{|l|}{ 4-Iodo } & \multicolumn{2}{|c|}{ Nonscaled } & \multicolumn{2}{|c|}{ Scaled } & \multirow[t]{2}{*}{ Observed } & \multirow[t]{2}{*}{ Assignment } \\
\hline Mode & Sym & AMl & PM.3 & AMI & PM3 & & \\
\hline 1 & $a^{\prime \prime}$ & 68 & 68 & 60 & 61 & & I wag + CCN wag \\
\hline 2 & $a^{\prime}$ & 119 & 124 & 106 & 110 & & $\mathrm{CI}$ bend $+C \mathrm{CN}$ bend \\
\hline 3 & $a^{\prime \prime}$ & 130 & 126 & 116 & 112 & & CCN wag \\
\hline 4 & $a^{\prime}$ & 1.31 & 143 & 116 & 127 & 146 & $\mathrm{CI}$ bend $+\mathrm{CCN}$ bend \\
\hline 5 & $a^{\prime \prime}$ & 228 & 221 & 203 & 297 & 231 & CI wag + CCN wag \\
\hline 6 & $a^{\prime}$ & 261 & 287 & 233 & 256 & & $\mathrm{CI}$ stretch $+\mathrm{CCN}$ bend \\
\hline 7 & $a^{\prime}$ & 274 & 296 & 244 & 263 & 252 & $\mathrm{CI}$ bend $+\mathrm{CCN}$ bend \\
\hline 8 & $a^{\prime \prime}$ & 376 & 364 & 335 & 324 & 377 & Ring torsion $+\mathrm{CCN}$ wag \\
\hline 9 & $a^{\prime \prime}$ & 4.33 & 405 & 385 & 360 & & $\mathrm{CCN}$ torsion + ring torsion $+\mathrm{CH}$ wag \\
\hline $\mathbf{0}$ & $a^{\prime}$ & 487 & 469 & 4.34 & 417 & 433 & Ring deformation \\
\hline 11 & $a^{\prime}$ & 576 & 541 & 513 & 481 & 478 & CCN bend \\
\hline 12 & $a^{\prime \prime}$ & 592 & 556 & 527 & 495 & 529 & $\mathrm{CCN}$ wag $+\mathrm{CH}$ wag \\
\hline 13 & $a^{\prime \prime}$ & 6.32 & 592 & 563 & 527 & 583 & $\mathrm{CCN}$ wag $+\mathrm{CH}$ wag \\
\hline 14 & $a^{\prime}$ & 662 & 639 & 589 & 569 & 592 & CCN bend + ring deformation \\
\hline 15 & $a^{\prime}$ & 716 & 673 & 637 & 599 & 629 & CCN bend \\
\hline 16 & $a^{\prime \prime}$ & 787 & 759 & 701 & 676 & 713 & $\mathrm{CCN}$ torsion $+\mathrm{CH}$ wag \\
\hline 17 & $a^{\prime}$ & 876 & 832 & 780 & 740 & 722 & Ring deformation \\
\hline 18 & $u^{\prime \prime}$ & 892 & 865 & 794 & 770 & 752 & $\mathrm{CH}$ wag \\
\hline 19 & $a^{\prime}$ & 942 & 946 & 839 & 842 & 8.36 & Ring deformation \\
\hline 20 & $a^{\prime \prime}$ & 958 & 953 & 853 & 848 & 844 & CH wag \\
\hline 21 & $a^{\prime \prime}$ & 996 & 994 & 886 & 885 & $9 ! 0$ & $\mathrm{CH}$ torsion \\
\hline 22 & $a^{\prime}$ & 1190 & 1126 & 1059 & 1002 & 1075 & CH bend \\
\hline 23 & $a^{\prime}$ & 1200 & 1143 & 1068 & 1017 & 1102 & $\mathrm{CH}$ bend \\
\hline 24 & $a^{\prime}$ & 1288 & 1200 & 1147 & 1068 & 1180 & $\mathrm{CH}$ bend \\
\hline 25 & $a^{\prime}$ & 1328 & 1274 & 1182 & 1134 & 1207 & CC stretch + CCN stretch \\
\hline 26 & $a^{\prime}$ & 1368 & 1.327 & 1217 & 1181 & 1267 & CC stretch \\
\hline 27 & $a^{\prime}$ & 1450 & 1428 & 1290 & 1271 & 1376 & Ring stretch \\
\hline 28 & $a^{\prime}$ & 1570 & 1564 & 1397 & 1392 & 1391 & CC stretch \\
\hline 29 & $a^{\prime}$ & 1654 & 16.34 & 1472 & 1454 & 1472 & CC stretch \\
\hline 30 & $a^{\prime}$ & 1753 & 1776 & 1560 & 1581 & 1546 & CC stretch \\
\hline 31 & $a^{\prime}$ & 1771 & 1795 & 1576 & 1597 & 1575 & CC stretch \\
\hline 32 & $a^{\prime}$ & 2560 & 2473 & 2278 & 2201 & 2233 & $C N$ stretch \\
\hline 33 & $a^{\circ}$ & 2560 & 2474 & 2278 & 2202 & 2238 & CN stretch (sym) \\
\hline 34 & $a^{*}$ & 3166 & 3055 & 2817 & 2719 & 2019 & $\mathrm{CH}$ stretch \\
\hline 35 & $a^{\prime}$ & 3168 & 306.3 & 2820 & 2726 & 3064 & $\mathrm{CH}$ stretch \\
\hline 36 & $a^{\prime}$ & 3181 & 3070 & 2831 & 2732 & 3095 & $\mathrm{CH}$ stretch \\
\hline
\end{tabular}

assignments proposed here for the halophthalonitriles are in good agreement with the work of Ramirez et al. [7] with due differences in experimental wave numbers, which depends on the substituency pattern, and the effect of heavy atom substituents. The high frequency vibrations of the $\mathrm{CH}$ stretching region and the $\mathrm{CN}$ stretching region are most readily assigned due to the high amplitude of the normal mode (low mode mixing) for the corresponding internal coordinate. For the molecules in this series all $\mathrm{CH}$ stretching vibrations were observed. It was of interest to follow the effect of a change in halosubstituent from bromine to iodine in the wavenumber value of fundamentals. For instance, an average downshift in wavenumber of $14 \mathrm{~cm}^{-1}$ is observed for $\mathrm{CH}$ stretching fundamentals upon a change in halosubstituent from bromine to iodine in analogous phthalonitriles. This mass effect is illustrated in Fig. 7. 
Table 6

Symmetry of vibration, AM1, PM3, experimental wavenumbers $(\mathrm{cm}$ '), and band assignments for 4.5-diiodophthalonitrile

\begin{tabular}{|c|c|c|c|c|c|c|c|}
\hline \multicolumn{2}{|c|}{ 4,5-1odo } & \multicolumn{2}{|c|}{ Nonscaled } & \multicolumn{2}{|l|}{ Scaled } & \multirow[t]{2}{*}{ Observed } & \multirow[t]{2}{*}{ Assignment } \\
\hline Mode & Sym & AMI & PM3 & AMl & PM3 & & \\
\hline 1 & $a_{2}$ & 44 & 41 & 39 & 37 & & $\mathrm{Cl}+\mathrm{CCN}$ wag \\
\hline 2 & $b_{1}$ & 74 & 90 & 66 & 80 & & CI wag \\
\hline 3 & $a_{1}$ & 94 & 95 & 84 & 84 & & $\mathrm{Cl}$ bend $+\mathrm{CCN}$ bend \\
\hline 4 & $a_{1}$ & 122 & 125 & 109 & 111 & & $\mathrm{Cl}$ bend $+\mathrm{CCN}$ bend \\
\hline 5 & $b$ & 123 & 131 & 109 & 116 & & $\mathrm{Cl}$ rock $+\mathrm{CCN}$ rock \\
\hline 6 & $a_{2}$ & 159 & 154 & 141 & 137 & & $\mathrm{CCN}$ wag + CI wag \\
\hline 7 & $b_{2}$ & 230 & 248 & 205 & 221 & & Cl stretch \\
\hline 8 & $h_{1}$ & 270 & 259 & 240 & 231 & 207 & $\mathrm{Cl}+\mathrm{CCN}$ wag \\
\hline 9 & $a_{1}$ & 301 & 342 & 268 & 304 & 255 & Cl stretch + ring deformation \\
\hline 10 & $b_{2}$ & 328 & 358 & 292 & 319 & 311 & $\mathrm{CCN}$ bend $+\mathrm{CI}$ bend \\
\hline 11 & $b_{1}$ & 414 & 398 & 369 & 354 & & Ring torsion + $\mathrm{CH}$ wag \\
\hline 12 & $a_{2}$ & 438 & 413 & 390 & 367 & 403 & CCN torsion + ring torsion \\
\hline 13 & $b_{2}$ & 23 & 528 & 465 & 470 & 480 & Ring deformation \\
\hline 14 & $a_{1}$ & 582 & 542 & 518 & 482 & 499 & CCN bend \\
\hline 15 & $b_{1}$ & 624 & 576 & 556 & 513 & 528 & $\mathrm{CCN}$ wag $+\mathrm{CH}$ wag \\
\hline 16 & $a_{2}$ & 687 & 660 & 612 & 587 & & Ring torsion \\
\hline 17 & $a_{1}$ & 705 & 685 & 627 & 610 & 619 & Ring deformation \\
\hline 18 & $b_{3}$ & 727 & 709 & 647 & 631 & 642 & CCN bend \\
\hline 19 & $a_{2}$ & 787 & 755 & 701 & 672 & 710 & CCN torsion \\
\hline 20 & $a_{\mathrm{t}}$ & 891 & 862 & 793 & 767 & 723 & Ring stretch + CCN stretch \\
\hline 21 & $b_{1}$ & 950 & 9.35 & 846 & 832 & 864 & $\mathrm{CH}$ wag \\
\hline 22 & $b_{3}$ & 963 & 957 & 857 & 852 & 885 & Ring deformation \\
\hline 23 & $a_{2}$ & 994 & 1039 & 884 & 925 & 906 & CH wag \\
\hline 24 & $a_{1}$ & 1205 & 1203 & 1072 & 1070 & 1098 & $\mathrm{CH}$ bend \\
\hline 25 & $b_{2}$ & 1273 & 1242 & 1133 & 1105 & 11.31 & $\mathrm{CH}$ bend \\
\hline 26 & $b_{2}$ & 1320 & 1271 & 1175 & 11.31 & 1217 & Ring deformation \\
\hline 27 & $a_{1}$ & 1360 & 1336 & 1210 & 1189 & 1264 & $C C$ stretch \\
\hline 28 & $a_{1}$ & I468 & 1468 & 1306 & 1306 & & Ring stretch \\
\hline 29 & $h_{2}$ & 1555 & 1538 & 1384 & 1369 & 1322 & $C C$ stretch \\
\hline 30 & $a_{1}^{2}$ & 1648 & 1652 & 1467 & 1470 & 1453 & CC stretch \\
\hline 31 & $a_{1}$ & 1749 & 1783 & 1556 & 1587 & 1523 & CC siretch \\
\hline 32 & $b_{2}$ & 1765 & 1786 & 1571 & 1589 & 1557 & CC stretch \\
\hline 33 & $b_{2}$ & 2560 & 2474 & 2278 & 2201 & 2232 & CN stretch \\
\hline 34 & $a_{1}$ & 2560 & 2475 & 2279 & 2202 & 2232 & CN stretch \\
\hline 35 & $b_{2}$ & 3163 & 3051 & 2815 & 2715 & 2991 & $\mathrm{CH}$ stretch \\
\hline 36 & $a_{1}^{2}$ & 3165 & 3052 & 2817 & 2716 & 3072 & $\mathrm{CH}$ stretch (sym) \\
\hline
\end{tabular}

Both the symmetric and antisymmetric $\mathrm{CN}$ stretching vibrations for the 4-substituted phthalonitriles were observed in the experimental spectra. For 3-iodophthalonitrile and the doubly substituted phthalonitriles the two modes are accidentally degenerate. Among similarly substituted phthalonitriles, a change from bromine to iodine substituent shows an average downshift in observed wave numbers of $4 \mathrm{~cm}^{-1}$. This lessened effect can be explained by the relative 'off of the ring' location of the $\mathrm{CN}$ bonds.
In the spectral region of the $\mathrm{C}=\mathrm{C}$ stretching modes, the aromatic ring vibrations overlap with the $\mathrm{C}-\mathrm{H}$ bending modes. The ring stretching modes showed a mass downshift smaller than that of the $\mathrm{CH}$ bending vibrations by a change in halosubstituent with an average decrease in wavenumber of 7 and $9 \mathrm{~cm}^{-1}$, respectively.

Out-of-plane wags and torsions and heavy substituent bends show average downshift of $7 \mathrm{~cm}$ i with change in halosubstituent. The effect is illustrated in Fig. 8. The mass effect was consistently 
observed across all four characteristic spectral regions, with stretching vibrations being most sensitive. At the same time the electronic effects were modest as indicated by the permanence of infrared intensities with the change in halosubstituent. A characteristic infrared intensity indicates relative invariance in $\left(\hat{\mu} \mu \hat{\rho} Q_{i}\right)$, which in turn is a qualitative indication of no significant change in the electronic structure with change in halosubstituent.

\subsection{Out-of-plane $\mathrm{C}-\mathrm{H}$ vibrations}

The identification of the $\mathrm{C}-\mathrm{H}$ wagging vibrational modes with a dipole moment derivative

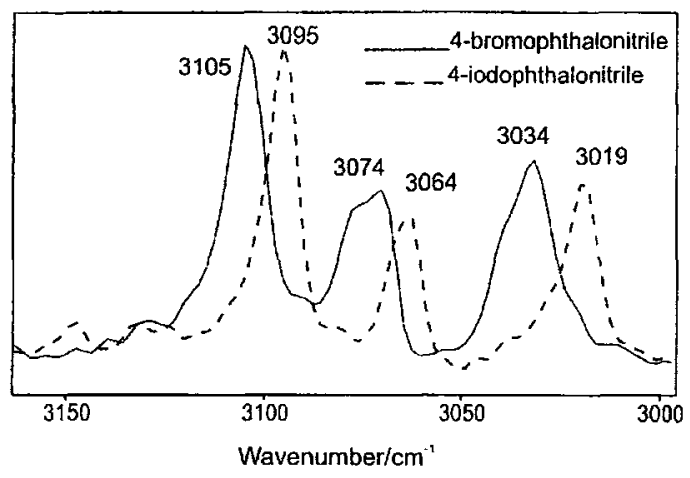

Fig. 7. C-H Stretching vibrations of 4-bromophthalonitrile and 4-iodophthalonitrile.

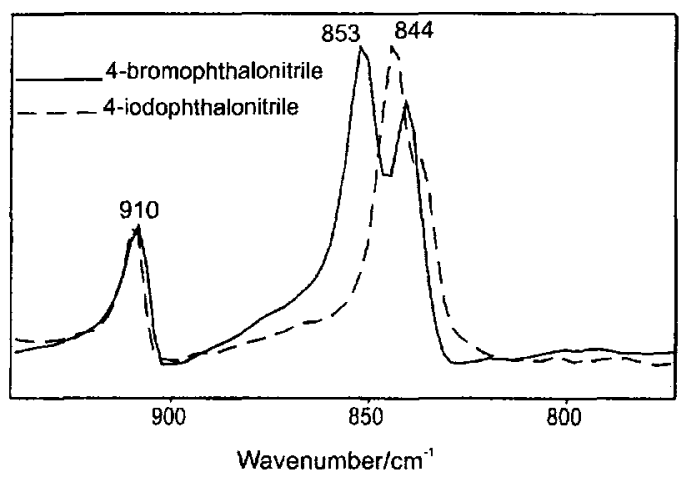

Fig. 8. Out-of-plane $\mathrm{C}-\mathrm{H}$ region of 4-bromophthalonitrile and 4-iodophthalonitrile. perpendicular to the molecular plane is of primary importance for studies of molecular organization in thin solid films of materials containing these molecular moieties. In 1,2-dicyanobenzene [7], the out-of-plane $\mathrm{C}-\mathrm{H}$ modes are assigned with wave numbers at 885,928 and $944 \mathrm{~cm}^{-1}$. In terephthalonitrile a strong infrared band at $846 \mathrm{~cm}$. was assigned to the wagging $\mathrm{C}-\mathrm{H}$ vibration [5]. In a series of four substituted benzonitriles Green and Harrison [25] have consistently observed two C-H out-of-plane vibrations: a weak infrared band in the $958-939 \mathrm{~cm}$ I region, and a strong infrared band in the $842.823 \mathrm{~cm}-1$ region. Similar bands with characteristic wavenumber and relative intensity in the infrared spectrum were observed in the series and have been assigned to the $\mathrm{C}-\mathrm{H}$ wagging. In the IR spectrum of 4-bromophthalonitrile a strong band at $853 \mathrm{~cm}{ }^{-1}$ has been assigned to the $\mathrm{C}-\mathrm{H}$ wagging of the two adjacent $\mathrm{C}-\mathrm{H}$ bonds. A second out-of-plane $\mathrm{C}$. $H$ is observed at $910 \mathrm{~cm}$. In 4,5-dibromophthalonitrile, where the there is no adjacent pair of $\mathrm{C}-\mathrm{H}$ bonds, only one wagging mode is observed at $915 \mathrm{~cm}^{-1}$ ' with strong infrared intensity. In the infrared spectrum of 3,6-dibromophthalonitrile the strong band of the wagging mode for the adjacent $\mathrm{C}-\mathrm{H}$ bond is observed at $847 \mathrm{~cm}^{-1}$. The infrared spectrum of the 3-iodophthalonitrile a strong band at $807 \mathrm{~cm} \quad$ is assigned to the $\mathrm{C}-\mathrm{H}$ wag. The high frequency wag is also observed at $860 \mathrm{~cm}^{-1}$. The 4-iodophthalonitrile follows the pattern observed for the 4-bromophthalonitrile (see Fig. 8) with a strong infrared band at 844 $\mathrm{cm}^{-1}$ and a weaker out-of-plane at $910 \mathrm{~cm}$. Finally, the 4,5-diiodophthalonitrile follows the template of the corresponding 4,5-dibromo derivative and the wagging at $906 \mathrm{~cm}{ }^{1}$ is observed. The strong lower wavenumber wag is not seen.

\section{Conclusions}

The infrared and FT-Raman spectra of a series of six novel halophthalonitriles have been studied. General assignments of observed fundamental vibrations have been proposed for all molecules in this study, on the basis of quantum chemical 
computations at the semi-empirical AMI and PM3 levels and computations at the SVWN DFT level. Encouraging results for vibrational properties were obtained using the DFT methods. The mass effect of halosubstitution (iodo for bromo) on fundamental vibrations was observed to be a downshift in wavenumber with a maximum of 14 $\mathrm{cm}^{-1}$. The out-of-plane $\mathrm{C}-\mathrm{H}$ vibrations have been identified in the infrared spectra of the series in consideration of their potential as vibrational probes for molecular organization studies in thin solid films.

\section{Acknowledgements}

Financial assistance from the Natural sciences and Engineering research council of Canada is gratefully acknowledged.

\section{References}

[1] P.J. Brach, S.J. Grammatica, O.A. Ossanna, L. Weinberger, J. Heterocyc. Chem. 7 (1970) 1403.

[2] J.A. Elvidge, R.P. Linstead, J. Chem. Soc. 3536 (1995)

[3] M.C. Castro-Pedrozo, G.W. King, J. Mol. Spectrosc. 73 (1978) 386.

[4] C.G. Barraclough, H. Bissett, P. Pitman, P.J. Thistlethwaite. Aust. J. Chent. 30 (1977) 753.

[5] J.F. Arenas, J.I. Marcos, F.J. Ramirez, Spectrochim Acta 44A (10) (1988) 1045.

[6] J.F. Arenas. J.I. Marcos, F.J. Ramirez, Can. J. Spectrose. 34 (1) (1989) 7.

[7] J.T. Lopez Navarrete, J.J. Quirante, M.A.G. Aranda, V.
Hernandez, F.J. Ramirez. J. Phys. Chem. 97 (1993) 10561.

[8] J. Hlorian, B.G. Johnson, J. Phys. Chem. 98 (1994) 3681.

[9] M.W. Wong, Chem. Phys. Lett. 256 (1996) 391.

[10] A. Berces, T. Ziegler. J. Chem. Phys. 98 (6) (1993) 4793.

[11] F. Campagna, A. Carotti, G. Cassini, Tetrahedron Lett. 21 (1977) 1813.

[12] C.C. Leznoff, D.S. Terekhov, C.R. McArthur, S. Vigh, J. Li, Can. J. Chem. 73 (1995) 435

[1.3] C.P. Rasmussen. J.F. Gardecki, J.N. Plampin, B.L. Twardzik, B.E. Reynolds. A.J. Molinari, N. Schwart?, W.W. Bennetts, B.E. Price. I.J. Markowski, J. Med. Chem. 21 (1978) I044.

[14] S.M. Marcuccio, P.I. Svirskaya, S. Greenburg, A.B.P. Lever, C.C. Leznoff, Can. J. Chem. 63 (1985) 3057.

[15] D.S. Terekhov, J.M.K. Nolan, C.R. McArthur, C.C. Leznoff, J. Org. Chem. 61 (9) (1996) 3034.

[16] W. Gottardi, Monatsh. Chem. 99 (1968) 815.

[17] D.S. Terekhov. Ph.D. Thesis, York University. Toronto. Ont., May 1997.

[18] M.J.S. Dewar, E.G, Zoebisch, E.F. Healy. J.J.P. Stewart, J. Am. Chent. Soc. 107 (1985) 3902.

[19] J.J.P. Stewart, J. Compul. Chem. I0 (1989) 209.

[20] M.J. Frisch, G.W. Trucks. M. Head-Gordon, P.M.W. Gill. M.W. Wong, J.B. Foresman, B.C. Johnson, H.B. Schlegel, M.A. Robb, E.S. Replogle, R. Gomperts, J.L. Andres, K. Raghavachari, J.S. Binkley, C. Gonzalez, R.L. Martin, D.J. Fox, D.J. DeFrees, J. Baker, J.J.P. Stewart, J.A. Pople, Gaussian 92. Revision E.1. Gaussian, PA, 1992

[21] J.C. Slater, The Self-Consistent Field for Molecules and Solids: Quantum Theory of Molecules and Solids, vol. 4. McGraw-Hill, New York. 1974.

[22] S.J. Vosko, L. Wilk, M. Nusail, Can. J. Phys. 58 (1980) 1200.

[23] Spartan IBM V.4.1.2GL, Wavefunction 18401, Von Karmen Suite 370. Irvinc, CA 92715.

[24] Y.N. Panchenko. Russ, Chem. Bull. 45 (4) (1996) 753.

[25] J.H.S. Green. D.J. Harrison. Spectrochim. Acta 32A (1976) 1279. 